\title{
CLASSIFICAÇÃO DOS PERIÓDICOS NO SISTEMA QUALIS DA CAPES - A MUDANÇA DOS CRITÉRIOS É URGENTE!
}

\author{
Classification of journals in the QUALIS System of CAPES - URGENT need of changing the \\ criteria!
}

Associação Médica Brasileira (AMB) preocupada com o futuro das publicações científicas brasileiras,
depois da divulgação dos novos critérios QUALIS da CAPES, organizou uma série de encontros
em sua sede em São Paulo. Os editores das principais revistas médicas do país, diretores da ABEC (Associação Brasileira de Editores Científicos) e os coordenadores das áreas de Medicina II e Medicina III da CAPES trocaram informações e, acima de tudo, elaboraram propostas para aprimorar o processo de avaliação dos periódicos científicos brasileiros pelo novo sistema QUALIS da CAPES. A produção científica classificada pelo QUALIS constituirá um dos itens principais da avaliação dos programas de pós-graduação no último triênio. Considerando que a principal fonte de artigos científicos para as revistas brasileiras são provenientes dos programas de pós-graduação vinculados à CAPES, era muito importante afinar o discurso e garantir uma linguagem comum para todas as partes envolvidas. Do lado dos editores há receio de que os novos critérios da CAPES possam criar uma subclasse de periódicos baseado exclusivamente no Fator de Impacto ISI. O critério anterior indicava, como ponto de corte, fator de impacto igual a 1 . Recentemente, algumas revistas brasileiras conseguiram, com enormes esforços, atingir este patamar. Entretanto, os novos critérios além de considerarem apenas o fator de impacto, estabeleceram pontos de corte bem mais elevados. Adotando-se esta medida, as revistas brasileiras passariam a ser preteridas pelos orientadores e alunos de pós-graduação - os grandes produtores da ciência brasileira - criando-se assim um círculo vicioso ao qual seria difícil a sobrevivência dos nossos periódicos.

Pelo lado da CAPES, falou o Professor João Pereira Leite, que além de coordenador da área de Medicina II, é também o atual representante da área de saúde no CTC - Conselho Técnico Científico - órgão máximo da CAPES. Este, durante uma de nossas reuniões, fez detalhada explanação sobre os critérios adotados nos triênios anteriores e o impacto dos mesmos sobre os Programas de pós-graduação do Brasil. Explicou ainda que, frente à evidente melhoria do nível dos programas era preciso elevar o ponto de corte ou de separação para melhor discriminá-los e estratificá-los qualitativamente. A partir dos dados provenientes dos programas - colhidos pelo sistema coleta CAPES - foi observado que muitos tinham mais de $50 \%$ - alguns mais de $80 \%$ - de sua produção científica publicada em periódicos dos estratos mais elevados. A CAPES por sua vez decidiu pela criação de um número maior de estratos para poder reclassificar os periódicos. Foi proposta uma escala decrescente segundo o valor do fator de impacto: A1, A2, B1, B2, B3, B4, B5 e C. Além disso, criou um fator de equivalência segundo o qual, a soma de publicações em revistas de estratos inferiores seria equivalente a um número menor de publicações dos estratos superiores. Assim, por exemplo, para uma determinada área, 2 artigos B1 equivaleriam a 1,2 artigo A1; 1 artigo B1 + 1 artigo A2 equivaleriam a 1,4 artigo A1; 3 artigos B2 equivalem a 1,2 A1. Segundo o professor João Leite "Essa equivalência beneficiaria as revistas com diferentes níveis de qualificação". O Professor Leite informou ainda que a nova classificação foi elaborada com base na mediana do fator de impacto das revistas, obtidas junto ao Journal Citation Report (JCR) e calculados anualmente pelo ISI Web of Knowledge. Para o cálculo da mediana foi elaborada uma lista das revistas em que cada área da CAPES publica. De posse desta lista, bem como dos respectivos fatores de impacto, foi calculada a mediana para cada área e construída a nova estratificação que varia entre $A 1, A 2, B 1$ até $B 5$ e $C$.

Os editores contrargumentaram o Professor Leite lembrando que a característica trienal do processo de avaliação CAPES conferiria um relativo descompasso para a reclassificação dos periódicos, a saber: várias revistas brasileiras terão seu fator de impacto aumentado ou publicado pela primeira vez ao longo de 2010, em especial as que acabaram de entrar para o ISI. Além disso, teriam que esperar três anos para mudar de categoria dentro do novo QUALIS! Outro questionamento dos editores diz respeito à escolha do fator 
de impacto publicado pelo Journal Citation Report (JCR) como ÚNICO e universal índice para aferição da qualidade dos periódicos. É grande o desvio-padrão dos valores dos fatores de impacto das revistas. Certamente por isso a CAPES utilizou a mediana destes índices para analisar o comportamento da produção dos programas de pós-graduação. De fato, segundo este critério, algumas especialidades médicas como as cirúrgicas, têm suas melhores revistas com fator de impacto mais baixo, o que poderia implicar num viés que lhes seria extremamente desfavorável.

Os dois lados concordaram que a valorização dos periódicos brasileiros é importante para o crescimento e desenvolvimento científico nacional. Para garantir e estimular este círculo virtuoso é preciso, entre outros, estimular e fomentar a citação de artigos de autores nacionais, intensificar os esforços de editores, revisores e autores dos periódicos para aumentar a qualidade dos artigos e, por outro lado, obter maior apoio dos órgãos governamentais, principalmente da CAPES e do CNPq, no que diz respeito, respectivamente, a administração de recursos financeiros e a estratificação qualitativa.

Os resultados destas discussões foram apresentados em diversos encontros de editores, coordenadores de Programas de pós-graduação e pesquisadores sendo complementados por novas sugestões. Que estas idéias, abaixo descritas, sirvam de conclusão para este editorial e que representem, ao mesmo tempo, uma ferramenta importante para a mudança dos critérios de classificação dos periódicos no sistema QUALIS da CAPES por parte dos organismos responsáveis. As propostas são as seguintes:

- A análise qualitativa dos periódicos brasileiros deve ser reavaliada e não envolver somente o Fator de Impacto publicado pelo Journal Citation Report (JCR).

- Devem ser consideradas e respeitadas as particularidades de cada área de interesse ou de cada especialidade.

- O parque editorial brasileiro, diferente do restante do mundo que é mantido basicamente pela iniciativa privada, é mantido à custa de universidades públicas e privadas e associações científicas de classe.
- Os periódicos brasileiros necessitam de maior apoio e incentivo, que poderão vir na forma de: bolsa para editores, apoio financeiro à publicação, maior visibilidade para os periódicos nacionais no exterior, critérios mais objetivos e abrangentes para classificação qualitativa, e apoio diferenciado $e$ correspondente ao desempenho de cada revista.

- Apoio à internacionalização dos periódicos científicos por meio de suporte para profissionalização do processo editorial e divulgação das revistas em outros países.

- Atualização contínua da classificação dos periódicos junto ao novo QUALIS sem precisar esperar pelo prazo da avaliação trienal.

- Participação de representantes de classe (ABEC, $A M B$, entre outros) no processo decisório junto ao sistema QUALIS da CAPES.

- Estímulo vigoroso à citação diretamente na fonte que são os programas de pós-graduação (por exemplo, determinando que programas de $P G$ notas 6 e 7, além de deverem obrigatoriamente ter uma porcentagem de publicações em revista de alto impacto, devam ter também cotas percentuais de publicações em periódicos nacionais. Com isto estarão contemplados os dois extremos da produção científica, pois os jovens e futuros pesquisadores iniciam sua carreira publicando em periódicos nacionais sob orientação de pesquisadores experientes.

Por fim, para corroborar todas estas ações e preocupada com o desenrolar das repercussões do novo QUALIS da CAPES e outras avaliações de periódicos, a ABEC (Associação Brasileira dos Editores Científicos) dedicou em seu último Encontro Nacional de Editores Científicos, realizado em novembro de 2009, três dias ao fórum de áreas. Neste, representantes da CAPES e editores de todas as áreas do conhecimento científico discutiram longamente 0 assunto e ao final propuseram as Diretrizes do Fórum de Áreas do XII Encontro Nacional dos Editores Científicos - 2009, que será oportunamente enviado a todas as agências brasileiras de fomento, e que deverá ocorrer periodicamente, pois, o processo é contínuo.

\section{Assinam este Editorial:}

Adagmar Andriolo

Aécio Flávio Meireles Souza

Alberto Queiroz Farias

Alfredo José Afonso Barbosa

Antonio Spina França Netto

Arnaldo José Hernandez

Aroldo F. Camargos

Benedito Barraviera

Bogdana Victoria Kadunc
Jornal Brasileiro de Patologia e Medicina Laboratorial

Revista GED

Revista Arquivos de Gastroenterologia

Jornal Brasileiro de Patologia e Medicina Laboratorial

Revista ARQUIVOS DE NEURO-PSIQUIATRIA

Revista Brasileira de Medicina do Esporte

Revista Femina

Journal of Venomous Animals and Toxins including Tropical Diseases

Surgical \& Cosmetic Dermatology da Soc. Brasileira de Dermatologia 
Bruno Caramelli

Carlos Eduardo Aguilera Campos

Carlos Brites

Dejair Caitano do Nascimento

Domingo M. Braile

Dov Charles Goldenberg

Edmund Chada Baracat

Edna T Kimura

Edson Marchiori

Eduardo de Paula Vieira

Eros Antônio de Almeida

Geraldo Pereira Jotz

Gilberto Camanho

Gilberto Friedman

Giovanni Guido Cerri

Ivomar Gomes Duarte

Izelda Maria Carvalho Costa

João Ferreira de Mello Júnior

Joel Faintuch

José Antônio Baddini Martinez

José Antonio Livramento

José Eduardo Ferreira Manso

José Luiz Gomes do Amaral

Linamara Rizzo Battistella

Luís dos Ramos Machado

Luiz Felipe P. Moreira

Luiz Henrique Gebrim

Marcelo Madeira

Marcelo Riberto

Marcus Bastos

Mário Cícero Falcão

Mario J. da Conceição

Mauricio Rocha e Silva

Milton Artur Ruiz

Milton K. Shibata

Mittermayer Barreto Santiago

Nelson Adami Andreollo

Osvaldo Malafaia

Regina Helena Garcia Martins

Renato Soibelmann Procianoy

Ricardo Baroudi

Ricardo Fuller

Ricardo Guilherme Viebig

Ricardo Nitrini

Rita Cristina Mainieri R. de Moura

Rogério Dedivitis

Ronaldo Damião

Sergio Lianza

Sigmar de Mello Rode

Winston Bonetti Yoshida

Zuher Handar
Revista da Associação Médica Brasileira

Revista Brasileira de Medicina de Família e Comunidade

Brazilian Journal of Infectious Diseases

Hansenologia Internationalis

Revista Brasileira de Cirurgia Cardiovascular

Revista Brasileira de Cirurgia Plástica

Revista da Associação Médica Brasileira

Arquivos Brasileiros de Endocrinologia e Metabologia

Revista Radiologia Brasileira

Revista Brasileira de Coloproctologia

Revista da Sociedade Brasileira de Clínica Médica

Revista Brasileira de Cirurgia Cabeça e Pescoço

Revista Brasileira de Ortopedia

Revista Brasileira de Terapia Intensiva

Radiologia Brasileira

Revista de Administração em Saúde

Anais Brasileiros de Dermatologia

Brazilian Journal of Otorhinolaryngology

Revista Brasileira de Nutrição Clínica

Jornal Brasileiro de Pneumologia

Revista Arquivos de Neuropsiquiatria

Revista do Colégio Brasileiro de Cirurgiões

Revista da Associação Médica Brasileira

Revista Acta Fisiátrica

Revista Arquivos de Neuropsiquiatria

Arquivos Brasileiros de Cardiologia

Revista Brasileira de Mastologia

Revista Brasileira de Mastologia

Revista Acta Fisiátrica

Jornal Brasileiro de Nefrologia

Revista Brasileira de Nutrição Clínica

Revista da Sociedade Brasileira de Anestesiologia

Revista Clinics

Revista Brasileira de Hematologia e Hemoterapia

Arquivos Brasileiros de Neurocirurgia

Revista Brasileira de Reumatologia

Arquivos Brasileiros de Cirurgia Digestiva

Arquivos Brasileiros de Cirurgia Digestiva

Brazilian Journal of Otorhinolaryngology

Jornal de Pediatria

Revista Brasileira de Cirurgia Plástica

Revista Brasileira de Reumatologia

Arquivos de Gastroenterologia

Dementia \& Neuropsychologia

Revista da Associação Brasileira de Medicina de Tráfego

Revista Brasileira de Cirurgia Cabeça e Pescoço

Urologia Contemporânea

Revista Medicina de Reabilitação

Brazilian Oral Research

Jornal Vascular Brasileiro

Revista Brasileira de Medicina do Trabalho 